\title{
Efecto del capital psicológico sobre las prácticas de liderazgo en pymes colombianas
}

\author{
Contreras, Francoise* \\ Juárez, Fernando**
}

\begin{abstract}
Resumen
El capital psicológico es un nuevo concepto que ha despertado un especial interés, debido a su efecto sobre el bienestar y la satisfacción laboral, factores que están relacionados con el desarrollo organizacional. El propósito de éste estudio fue describir el capital psicológico de un grupo de directivos de PYMES colombianas y determinar si éste ejerce un efecto sobre sus prácticas de liderazgo. Para medir las variables que constituyen el capital psicológico se aplicó el Test de Orientación Vital Revisado (LOT-R), la Escala de Autoeficacia Generalizada [EAG], la Escala de Esperanza Estado [SHS] y la Escala de Resiliencia [RS]. Las prácticas de liderazgo se evaluaron a través del Inventario de Prácticas de Liderazgo (IPL). De acuerdo con los resultados, los participantes presentaron un alto nivel de capital psicológico, en el que el optimismo obtuvo la menor puntuación. El capital psicológico, como constructo global, influyó en las características de liderazgo, pero no de igual forma en todas las prácticas estudiadas. A nivel individual, excluyendo al optimismo, las demás variables del capital psicológico influyeron en las prácticas de liderazgo, también de forma diferencial.
\end{abstract}

Palabras clave: Capital psicológico, liderazgo, esperanza, resiliencia, optimismo.

Recibido: 15-06-11. Aceptado: 05-04-13

* Psicóloga, Ph.D. en Psicología, Especialista y Magíster en Educación, Profesora Titular de la Universidad del Rosario, Bogotá, directora de la Línea de Liderazgo del Grupo de Investigación en Perdurabilidad Empresarial (GIPE) de la Facultad de Administración, e-mail: francoise.contreras@urosario.edu.co

** Psicólogo, Ph.D en Psicología. Profesor Asociado de la Universidad del Rosario, Bogotá, miembro del Grupo de Investigación en Perdurabilidad Empresarial (GIPE) de la Facultad de Administración, e-mail: fernando.juarez@urosario.edu.co 


\title{
The Effect of Psychological Capital on Leadership Practices in Colombian SMEs
}

\begin{abstract}
Psychological capital is a new concept that has awakened a special interest, due to its effect on well-being and satisfaction in the workplace, factors that are related to organizational development. The purpose of this study was to describe the psychological capital of a group of Colombian SME directors and determine if this exercises an effect on their leadership practices. To measure the variables that make up psychological capital, the Life Orientation Test, Revised (LOT-R), the Scale of Generalized Self-Efficacy (Escala de Autoeficacia Generalizada-EAG), the State Hope Scale (SHS) and the Resilience Scale (RS) were applied. Leadership practices were evaluated using the Inventory of Leadership Practices (Inventario de Prácticas de Liderazgo-IPL). According to the results, participants evidenced a high level of psychological capital in which optimism obtained the highest score. Psychological capital, as a global construct, influenced leadership characteristics, but not in the same way for all the practices studied. On the individual level, excluding optimism, the other psychological capital variables influenced the leadership practices, also in a differential manner.
\end{abstract}

Key words: Psychological capital, leadership, hope, resilience, optimism.

\section{Introducción}

En las últimas décadas, los estudios respecto al efecto de los estilos de liderazgo sobre el bienestar de los subordinados y su relación con el desempeño laboral, se han incrementado considerablemente. Actualmente, existe suficiente evidencia para afirmar que determinados estilos de liderazgo guardan relación con el nivel de satisfacción laboral (Chiok Foong Loke, 2001), el estrés percibido (Bratt et al., 2000), la motivación al logro (Mc Neese-Smith, 1999), el clima organizacional, e incluso con la cultura empresarial, entre muchos otros aspectos. Estos estudios en su mayoría, asumen el liderazgo desde la perspectiva de las prácticas de dirección, y de la relación que los directivos establecen con sus colaboradores. Se ha encontrado por ejemplo que el acceso a la información, el empoderamiento, las oportunidades de desarrollo y un adecuado clima organizacional inciden favorablemente sobre la satisfacción laboral (Upenieks, 2003), mientras que la imposibilidad para participar en la toma de decisiones y la falta de apoyo organizacional percibido, afectan no solo la satisfacción laboral, sino también la calidad del desempeño, el nivel de compromiso y la capacidad de innovación de los trabajadores (Eisenberger et al., 1990).

La investigación se llevó a cabo pequeñas y medianas empresas (pymes) de la ciudad de Bogotá, Colombia; se trabajó con una muestra por disponibilidad de 94 directivos cursantes de estudios de postgrado en áreas empresariales, su participación fue voluntaria y anónima. Se aplicaron instrumentos para la recopilación de la información empírica, entre ellos: Test de Orientación Vital Revisado (LOT-R), Escala de Autoeficacia Generalizada (EAG), Escala de Esperanza-Estado (SHS), Escala de Resilencia (RS) y el 
Inventario de Prácticas de Liderazgo (IPL); luego de las aplicaciones colectivas por grupos de aproximadamente 20 personas, con aplicaciones de 30 minutos por grupos. Para esto, se utilizó un diseño transversal, descriptivo y correlacional de influencia. En primer lugar, se describió el capital psicológico, así como sus componentes junto con las prácticas de liderazgo, y posteriormente, se observó si existía relación entre ellas, y si las variables del capital psicológico influyen en las prácticas de liderazgo.

\section{Liderazgo y bienestar laboral}

Atendiendo a la clasificación de los estilos de liderazgo, existe una amplia literatura que sustenta que algunos comportamientos del líder incluidos dentro del llamado liderazgo transformacional, se han asociado con el nivel de satisfacción laboral (Dunham-Taylor, 2000), en cuanto logran inspirar y motivar a los trabajadores, lo que posibilita el que ellos, le encuentren significado a su trabajo y sentido a lo que hacen (Sparks y Schenk, 2001).

Pirela (2010) describe éste liderazgo como un proceso caracterizado por altos niveles de moralidad y motivación donde los líderes, llamados transformadores, pretenden incrementar el grado de conciencia de sus colaboradores, a través de ideales y valores como la libertad, la justicia y el humanitarismo. Este tipo de liderazgo afirma, debe ser visto como un proceso de influencia a nivel micro (entre Individuos) y a nivel macro (movilización de poder con miras a cambiar sistemas sociales y producir reformas institucionales). Consecuentemente, otros autores han encontrado que éste tipo de liderazgo disminuye la posibilidad de aparición de síndromes relacionados con el estrés laboral como el burnout (Sosik y Godshalk, 2000).

Con base en éstos y muchos otros estudios, se puede afirmar sin lugar a dudas, que los estilos de liderazgo y la calidad de las relaciones interpersonales que los caracterizan, ejercen un efecto importante sobre los empleados, y que en este orden de ideas, los directivos tienen potencialmente la posibilidad de incrementar o disminuir los estándares de calidad de vida de sus trabajadores, entendida ésta, como una percepción del sujeto.

Dado que el comportamiento está mediado por la cognición, cabría suponer la existencia de variables intraindividuales (creencias, experiencias previas, expectativas, afectividad, estilos atribucionales, entre otros) que pueden estar determinando los estilos de liderazgo, y sus correspondientes comportamientos asociados, lo cual está siendo recientemente estudiado. Aún se tiene escaso conocimiento sobre cuáles son los factores individuales, que pueden ejercer un efecto sobre la forma en que los líderes se relacionan con sus colaboradores y las características propias del sujeto, que están incidiendo sobre sus prácticas de liderazgo.

\section{Capital psicológico y liderazgo}

Entre las variables cognitivas estudiadas están aquellas que conforman el capital psicológico (Luthans et al., 2007) el cual hace parte del llamado comportamiento organizacional positivo (Luthans, 2002), que surge de la teoría e investiga- 
ción en psicología positiva, aplicada al contexto laboral (Seligman y Csikszentmihalyi, 2000). El capital psicológico es definido como un estado positivo de desarrollo caracterizado por cuatro variables: 1) autoeficacia, 2) optimismo, 3) esperanza, y 4) resiliencia (Luthans, Youssef y Avolio, 2007).

La autoeficacia o confianza en sí mismo, hace referencia a la convicción de un individuo acerca de sus habilidades para movilizar la motivación, los recursos cognitivos y las acciones necesarias para lograr exitosamente una tarea determinada (Stajkovic y Luthans, 1998). El optimismo, alude a la atribución interna, relativamente estable y global con respecto a eventos positivos (Seligman, 1998), son atribuciones sobre éxitos actuales y futuros (Luthans et al., 2007), basadas en evaluaciones objetivas y realistas de lo que se puede lograr en una situación dada (Luthans et al., 2008).

La esperanza por su parte, es definida como un estado emocional positivo que involucra un sentido de éxito, voluntad, planeación y acción para lograr las metas, implica el perseverar para alcanzar los objetivos y redireccionar las estrategias cuando sea necesario (Snyder, 2000); en este sentido el constructo de esperanza está básicamente orientado hacia el logro de metas y tiene dos componentes previamente establecidos que son: 1) los pensamientos orientados hacia el establecimiento de alternativas para lograr las metas (pathways) y, 2) los pensamientos orientados hacia la acción (agency) (Snyder, 1994; Snyder et al., 1991).

Finalmente, la resiliencia aplicada al contexto laboral se ha descrito como la capacidad psicológica de recuperarse ante la adversidad, la incertidumbre, el conflicto, el fracaso e incluso de eventos altamente positivos, como adquirir nuevas responsabilidades en cargos de mayor exigencia y responsabilidad (Luthans, 2002), lo que requiere adecuadas respuestas de afrontamiento, para adaptarse e incluso salir fortalecido (Luthans et al., 2007).

Los estudios sobre capital psicológico en las organizaciones son muy recientes y se han orientado principalmente hacia el efecto en el desempeño que tiene el que los trabajadores que lo posean. Al respecto, se ha encontrado que el capital psicológico se ha asociado con el nivel de desempeño, bienestar y satisfacción laboral (Luthans et al., 2007; Luthans et al., 2005; Peterson y Luthans, 2003), con el comportamiento ciudadano, mayor identificación y compromiso del trabajador con la organización, menor frecuencia de conductas problemáticas en el lugar de trabajo (Norman et al., 2010; Youssef y Luthans, 2007) y mejor manejo del estrés laboral (Avey, Luthans y Jensen, 2009). Por el contrario, los trabajadores con bajo nivel de capital psicológico, se muestran más despreocupados, tienen mayor tendencia a abandonar el cargo y suelen presentar más conductas disruptivas que afectan sus relaciones interpersonales (Avey et al., 2010).

En éste mismo orden de ideas, si se observan las características del capital psicológico de forma independiente se ha encontrado que el optimismo y la autoeficacia se han relacionado con el desempeño laboral (Luthans et al., 2005; Seligman, 1998), y con la satisfacción/felicidad (Youssef y Luthans, 2007), la resiliencia por su parte, se ha asociado con la actitudes favorables hacia el trabajo y el compromiso del trabajador (Youssef y Luthans, 2007). 
No obstante, existe evidencia empírica a través de múltiples muestras que indican que los empleados que poseen los cuatro componentes del capital psicológico presentan un mejor desempeño, y presentan más altos índices de satisfacción que cuando tienen solo uno de los cuatro componentes de manera individual (Luthans et al., 2007). Lo anterior demuestra no solo la utilidad del constructo sino que cada uno de los componentes del capital psicológico son conceptual y psicométricamente distintos entre sí, lo que incrementa su validez (Luthans et al., 2008). Debido a lo anterior, varios autores sostienen que las variables del capital psicológico deben verse como un continuo (Avey, Luthans y Mhatre, 2008) y que éstas pueden ser desarrolladas mediante el aprendizaje (Seligman y Csikszentmihalyi, 2000).

A través de estudios recientes se ha demostrado la relación entre el capital psicológico y el liderazgo transformacional (Toor y Ofori, 2010), y se ha evidenciado también el efecto de esta asociación sobre la calidad del desempeño (Gooty et al., 2009). No obstante, algunos autores consideran que es la confianza en la gestión, característica de estos estilos de liderazgo, la que media la relación entre el capital psicológico y el desempeño laboral (Clapp-Smith, Vogelgesang y Avey, 2009). Al parecer es la confianza en los líderes, unido a la percepción de eficacia personal la que favorece el desempeño laboral (Jiayan, Oi-Ling y Kan, 2010), aspecto que debe ser más ampliamente estudiado.

Es así como el liderazgo transformacional juega un rol mediador en el capital psicológico (efectividad, esfuerzos adicionales y satisfacción) (Toor y Ofori, 2010). Las prácticas asociadas a este estilo de liderazgo, es decir, apertura y claridad en su comportamiento, compartir información para la toma de decisiones, aceptar sugerencias y proporcionar retroalimentación constructiva, entre otras, inciden en que los seguidores tiendan a tener mayor eficacia personal, esperanza, optimismo y resiliencia, es decir, desarrollen un mayor capital psicológico (Walumbwa et al., 2011).

De acuerdo con lo anterior, es evidente que el capital psicológico constituye un factor de desarrollo humano y empresarial de gran importancia. Luthans y Youssef (2004) afirman que éste factor podría incluso convertirse en un factor de sostenibilidad para lograr una ventaja competitiva en la economía global actual. Para estos autores, el capital psicológico constituye una nueva dimensión de los recursos humanos, junto con el capital humano (conocimiento explícito y tácito) y el capital social (redes, normas/valores y confianza). El considerar el capital psicológico como una dimensión más, tendrá importantes implicaciones; en cuanto involucra capacidades psicológicas susceptibles de ser medidas y desarrolladas para favorecer el desarrollo organizacional.

No obstante, a pesar de su creciente interés y reconocimiento, el capital psicológico aún no ha recibido suficiente atención por parte de académicos del área de los negocios ni por parte de los empresarios. Respecto a éstos últimos, esto puede deberse a la tendencia que tienen los directivos de centrarse más en el cambio de estrategias para un mejor manejo del recurso humano, que preocuparse por las deficiencias o asuntos per- 
sonales relacionados con sus trabajadores, ignorando la posibilidad potencial que tienen, como líderes, de incidir sobre la confianza, la esperanza, el optimismo y la resiliencia, y así mejorar tanto el desarrollo personal como el desempeño organizacional (Luthans, Luthans y Luthans, 2004).

A nivel académico, aún quedan muchos aspectos por aclarar sobre la utilidad de aplicar este constructo a distintos elementos que hacen parte del contexto empresarial, en relación con los individuos y con los procesos que configuran a las organizaciones. No es claro por ejemplo, el papel mediador del capital psicológico (ya sea como constructo integral o de forma individual) sobre las prácticas de liderazgo que ejercen los directivos. El propósito de éste estudio es precisamente determinar si el capital psicológico de los directivos guarda relación con los comportamientos de liderazgo, o si existen componentes de éste constructo que de manera individual estén ejerciendo un efecto más determinante que esos comportamientos.

Con base en lo anterior, se plantean dos hipótesis:

1. El capital psicológico incide en las prácticas de liderazgo de los directivos de las PYMES estudiadas.

2. Existen componentes independientes del capital psicológico que influyen sobre las prácticas de liderazgo.

\section{Capital psicológico y prácticas de liderazgo en pymes colombianas}

Se evalúo el capital psicológico de 94 directivos de PYMES en la ciudad de
Bogotá, Colombia. Para realizar esta evaluación, se seleccionó un conjunto de instrumentos de manera que tuvieran excelentes propiedades psicométricas de confiabilidad y validez, que existiera una versión en español y que ya hubieran sido aplicados a población de habla hispana. A continuación se describirá cada uno de ellos con sus características.

\subsection{Test de Orientación Vital Re-} visado (LOT-R): Instrumento constituido por cinco opciones de respuesta que permite evaluar el optimismo disposicional o las expectativas de los individuos acerca de que puedan ocurrirles resultados favorables en el futuro. La prueba consta de seis ítems y otros cuatro ítems más, neutros. El instrumento ha demostrado una consistencia interna de 0.78 y una correlación con la versión amplia del LOT original de 0.95, el índice de fiabilidad fue de 0.67 (Scheier, Carver y Bridges, 1994). Este instrumento ha sido aplicado y validado en población de habla castellana donde se ha encontrado una confiabilidad de 0.68 en una muestra de estudiantes universitarios españoles (Ferrando, Chico y Tous, 2002).

\subsection{Escala de Autoeficacia Ge-} neralizada (EAG): Este instrumento consta de 10 ítems y fue diseñado para medir este constructo a nivel de disposición general de la personalidad. La escala fue elaborada originalmente en alemán por Jerusalem y Schwarzer en 1981 (Jerusalem y Schwarzer, 1992), utilizada y validada en numerosos estudios (Schwarzer et al., 1997) y adaptada a varios idiomas, entre ellos el chino y el español, alcanzando una alta consistencia interna (coeficiente Alpha de Cronbach de entre 0.79 y 0.93 ) y un alto grado de vali- 
dez convergente y discriminante. En este estudio, se utilizó la versión en español hecha por Baessler en Costa Rica (Baessler y Schwarzer, 1996). Estudios posteriores realizados por Juarez y Contreras (2008), demuestran que el instrumento puede ser aplicado en población colombiana, dado que demostró adecuadas propiedades psicométricas

\subsection{Escala de Esperanza Estado}

(SHS): Esta escala fue desarrollada y validada por Snyder et al. (1996), y evalúa la esperanza como un estado. La escala consta de 6 ítems con ocho alternativas de respuesta que van desde definitivamente falso hasta definitivamente cierto. La escala proporciona a su vez, dos subescalas, una orientada hacia las alternativas para lograr las metas (pathways) y la otra hacia la acción para lograrlas (agency). El total se obtiene incluyendo estas dos subescalas, cuyo resultado estará en el rango de 6 a 48. El instrumento ha demostrado adecuada confiabilidad interna tanto para la escala total (Alpha de Conbrach de 0.90 a 0.95) como para sus subescalas (Alpha de Conbrach de 0.90). Así mismo posee una adecuada estructura factorial y validez discriminante (Feldman y Snyder, 2000; Snyder et al., 1996).

4.4. Escala de Resiliencia (RS): Esta escala fue desarrollada por Wagnild y Young (1993), para evaluar las cualidades personales que favorecen la adaptación individual resiliente. Se utilizó la versión en castellano de Heileman, Lee y kury (2003), quienes encontraron una correlación negativa entre resiliencia y síntomas depresivos. La escala que ha demostrado alta consistencia interna (alfa de Cronbach de 0.93 ), consta de 25 ítems con 7 alternativas de respuesta que van desde totalmente en desacuerdo, hasta totalmente de acuerdo. La RS evalúa la resiliencia a través de dos factores: 1) competencia personal, entendida como el reconocimiento de la capacidad personal, independencia, dominio y perseverancia, entre otros, y 2) aceptación de sí mismo y de la vida como sinónimo de adaptación y flexibilidad. El primer factor está compuesto por 17 ítems y el segundo por 8 . El puntaje total se obtiene sumando los puntajes obtenidos en los factores anteriormente enunciados y los resultados se ubican en un rango que va de 25 a 175. Los resultados obtenidos permiten ubicar al sujeto en alto (valores mayores a 147), moderado (valores entre 121 y 146) o escaso (valores menores a 121) nivel de resiliencia.

4.5. Inventario de Prácticas de liderazgo (IPL): Se utilizó la versión adaptada por Robles, De la Garza y Medina (2008), quienes a su vez, utilizaron la primera adaptación desarrollada por Mendoza (2005), del inventario original Leadership Practices Inventory [LPI] desarrollado por Kouzes y Posner (1988, 2003). El propósito del instrumento es evaluar el estilo de liderazgo a través de cinco comportamientos: 1) desafiar los procesos y extender los riesgos, 2) inspirar una visión compartida, 3) habilitar a los demás para que actúen, 4) modelar el camino y, 5) dar aliento al corazón. Esta última dimensión es la única que pertenece al liderazgo transaccional, las otras cuatro corresponden al liderazgo transformacional. La escala está constituida por 30 ítems, con cinco alternativas de respuesta. El Alpha de Cronbach para la escala en general fue mayor a .70 lo que de- 
muestra que la escala utilizada es confiable. Así mismo, cada una de las escalas ha demostrado ser confiable, encontrándose los siguientes Alpha de Conbach: $1)$ desafiar los procesos $(0.72), 2)$ inspirar una visión compartida $(0.80), 3)$ habilitar a los demás para que actúen (0.76), 4) modelar el camino (0.83), y 5) dar aliento al corazón (0.75) (Robles et al., 2008).

Al aplicar las escalas anteriormente descritas en pymes colombianas, se obtienen los resultados; en la Tabla 1 se muestran las características sociodemográficas de los participantes, quienes en su mayoría fueron mujeres (mujeres $=63,67 \%$, hombres $=31,33 \%$ ), con una edad promedio de 34 años, soltero/as ( $n=44,46.8 \%)$ o casada/os ( $n=33,35.1 \%$ ), con un estrato socioeconómico de tres $(n=35,37.2 \%)$ o cuatro ( $n=36,38.3 \%$ ), lo que en Colombia corresponde a un nivel de ingresos y de vivienda medio y medio-alto respectivamente, siendo el máximo estrato el seis.

Los participantes eran trabajadores independientes ( $n=65,69.1 \%)$, cursando estudios de especialización ( $\mathrm{n}=$ $48,51.1 \%$ ) o maestría ( $n=46,48.9 \%$ ). Las empresas donde, principalmente, se desempeñaban como coordinadores/as ( $n=40,42.6 \%)$ o directora/es ( $n=24$, $25.5 \%$ ), desde hacía menos de tres años ( $n=39,41.5 \%)$ o de tres a cinco años ( $n$ $=35,37.2 \%$ ), pertenecían principalmente al sector servicios ( $n=59,62.8 \%)$ y tenían, generalmente, menos de $11(\mathrm{n}=$ $56,59.5 \%)$ o entre 11 y 50 ( $n=26$, $27.7 \%$ ) empleados a cargo. No obstante, los participantes podían tener a su cargo de 51 a 200 empleados ( $n=63,67 \%$ ), debido al elevado número de personas que realizaban actividades para algunas empresas a través de agencias de servi- cios. De este modo, en el estudio predominaron las empresas pequeñas y medianas, perteneciendo los participantes a PYMES de distintos sectores de la economía nacional.

En términos generales, los resultados muestran un alto nivel de capital psicológico en este grupo de directivos. En el Gráfico 1 se puede observar el porcentaje obtenido en cada una de las variables que configuran el constructo, considerando el rango máximo posible en cada una de ellas.

En la Tabla 2 pueden observarse a nivel descriptivo, los resultados obtenidos en cada una de las variables estudiadas, tanto para capital psicológico como para las prácticas de liderazgo.

Mediante modelos de regresión, se observó el efecto de las dimensiones del capital psicológico sobre las prácticas de liderazgo existentes en el IPL. Antes de aplicar dichos modelos de regresión, se obtuvo la matriz de correlaciones entre las escalas y subescalas de los diferentes instrumentos, mostrada en la Tabla 3. Di-

\section{Gráfico 1 \\ Capital psicológico de los directivos en pymes colombianas}

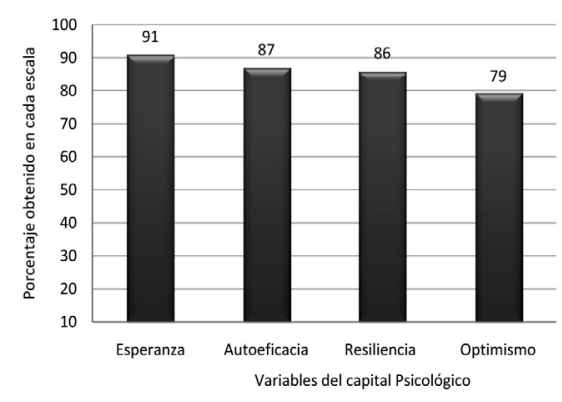

Fuente: Elaboración propia. 


\section{Tabla 1}

\section{Características sociodemográficas de los participantes}

\begin{tabular}{|c|c|c|c|}
\hline & $\bar{X}(\mathrm{DE})$ & & \\
\hline \multirow[t]{2}{*}{ EDAD } & $34.04(7.7)$ & & \\
\hline & $\mathrm{n}(\%)$ & & $\mathrm{n}(\%)$ \\
\hline SEXO & \multicolumn{3}{|c|}{ EMPLEADOS EN LA EMPRESA } \\
\hline Hombres & $31(33.0)$ & Menos de 11 & $56(59.5)$ \\
\hline Mujeres & $63(67.0)$ & De 11 a 50 & $26(27.7)$ \\
\hline ESTADO CIVIL & & De 51 a 200 & $8(8.5)$ \\
\hline Soltero & $44(46.8)$ & Más de 200 & $4(4.3)$ \\
\hline Casado & $33(35.1)$ & SECTOR ECONÓMICO & \\
\hline Unión libre & $12(12.8)$ & Industrial & $7(7.4)$ \\
\hline Divorciado & $5(5.3)$ & Comercial & $6(6.4)$ \\
\hline ESTRATO SOCIOECONÓMICO & & Servicios & $59(62.8)$ \\
\hline 1 & $1(1.1)$ & Otros & $22(23.4)$ \\
\hline 2 & 1(1.1) & CARGO & \\
\hline 3 & $35(37.2)$ & Presidente/Gerente & 3(3.2) \\
\hline 4 & $36(38.3)$ & Director & $24(25.5)$ \\
\hline 5 & $14(14.9)$ & Coordinador & $40(42.6)$ \\
\hline 6 & $7(7.4)$ & Supervisor & $8(8.5)$ \\
\hline TRABAJO & & Otro & $19(20.2)$ \\
\hline Empresario & 4(4.3) & ANTIGÜEDAD EN EL CARGO & \\
\hline Trabajo independiente & $65(69.1)$ & Menos de 3 años & $39(41.5)$ \\
\hline Trabajo dependiente & 11(11.7) & De 3 a 5 años & $35(37.2)$ \\
\hline Otros & $14(14.9)$ & De 6 a 10 años & $12(12.8)$ \\
\hline EDUCACIÓN & & Más de 10 años & $8(8.5)$ \\
\hline Cursando Especialización & $48(51.1)$ & EMPLEADOS CARGO & \\
\hline \multirow[t]{3}{*}{ Cursando Maestría } & $46(48.9)$ & Menos de 11 & 18(19.2) \\
\hline & & De 11 a 50 & $13(13.8)$ \\
\hline & & De 51 a 200 & $63(67.0)$ \\
\hline
\end{tabular}

Fuente: Elaboración propia.

cha matriz, está constituida por los coeficientes de correlación Rho de Spearman, debido a que las distribuciones carecían de normalidad. En general se observan correlaciones moderadas, pero significativas, entre las diferentes dimensiones; las prácticas de liderazgo se relacionan con todas las otras dimensiones de manera significativa, oscilando los coeficien- tes de correlación significativos entre 0.229 (Modelar-Optimismo) y 0.678 (Inspirar-Resiliencia, Competencia personal). La única dimensión que no muestra ninguna correlación significativa con las prácticas de liderazgo es ResilienciaAceptación de la vida y de sí mismo.

Por otra parte, las prácticas de liderazgo se relacionan entre sí, lo que resul- 
Efecto del capital psicológico sobre las prácticas de liderazgo en pymes colombianas Contreras, Francoise y Juárez, Fernando

Tabla 2

Capital psicológico y estilos de liderazgo

\begin{tabular}{lccccc}
\hline & Media & Mediana & $\begin{array}{c}\text { Desviación } \\
\text { Estándar }\end{array}$ & Mínimo & Máximo \\
\hline $\begin{array}{l}\text { Autoeficacia (EAG) } \\
\quad \text { Autoeficacia }\end{array}$ & 34.96 & 35.50 & 3.87 & 21 & 40 \\
$\begin{array}{l}\text { Optimismo (OP) } \\
\quad \text { Optimismo }\end{array}$ & 19.03 & 19.00 & 2.78 & 12 & 24 \\
$\begin{array}{l}\text { Esperanza (SHS) } \\
\quad \text { Esperanza-Sustantividad }\end{array}$ & 21.90 & 22.50 & 2.38 & 12 & 24 \\
$\quad$ Esperanza-Vías & 21.87 & 22.50 & 2.77 & 6 & 24 \\
$\quad$ Esperanza-Total & 43.78 & 45.00 & 4.65 & 18 & 48 \\
$\quad$ Resiliencia (RE) & & & & & \\
$\quad$ Resiliencia-Competencia personal & 105.89 & 107.00 & 8.45 & 81 & 118 \\
$\quad$ Resiliencia-Aceptación de sí mismo & & & & & \\
$\quad$ y de la vida & 44.31 & 45.00 & 5.14 & 29 & 56 \\
Estilos de liderazgo (IPL) & & & & & \\
$\quad$ Modelar & 26.16 & 26.00 & 2.49 & 20 & 30 \\
$\quad$ Inspirar & 23.81 & 24.00 & 3.41 & 14 & 30 \\
$\quad \begin{array}{l}\text { Desafiar } \\
\text { Habilitar }\end{array}$ & 24.02 & 24.00 & 3.28 & 16 & 30 \\
$\quad$ Alentar & 25.34 & 25.00 & 2.54 & 19 & 30 \\
\hline
\end{tabular}

Fuente: Elaboración propia.

ta esperable debido a que forman parte del mismo constructo, observándose coeficientes de correlación de entre 0.50 y ligeramente superiores a 0.60 . A su vez las otras dimensiones correlacionan también entre sí con coeficientes de entre 0.30 y 0.50 . De esta manera, las diferentes escalas y subescalas de los instrumentos se relacionan con coeficientes moderados.

Las correlaciones de las dimensiones del capital psicológico con las diferentes prácticas de liderazgo, indican que un modelo de regresión puede ser apropiado. Debido a la ausencia de normalidad mencionada, se utilizaron modelos de regresión ordinal para determinar la in- fluencia de las variables de capital psicológico sobre las diferentes prácticas de liderazgo.

En la Tabla 4, se muestran los diferentes modelos de regresión utilizados, los cuales resultaron significativos para todos los tipos de prácticas. En el modelo 1 (variable dependiente del IPL: Modelar) la regresión resultó significativa (Chi cuadrado $=168.517, p=.000$ ) explicando una varianza del $83 \%$. En el modelo 2 (Variable dependiente del IPL: Inspirar) la regresión resultó significativa (Chi cuadrado $=479.853, p=.000$ ) explicando una varianza del $99 \%$. En el modelo 3 (Variable dependiente del IPL: Desafiar) la regresión resultó significativa (Chi cuadra- 
Revista Venezolana de Gerencia, Año 18, No. 62, 2013

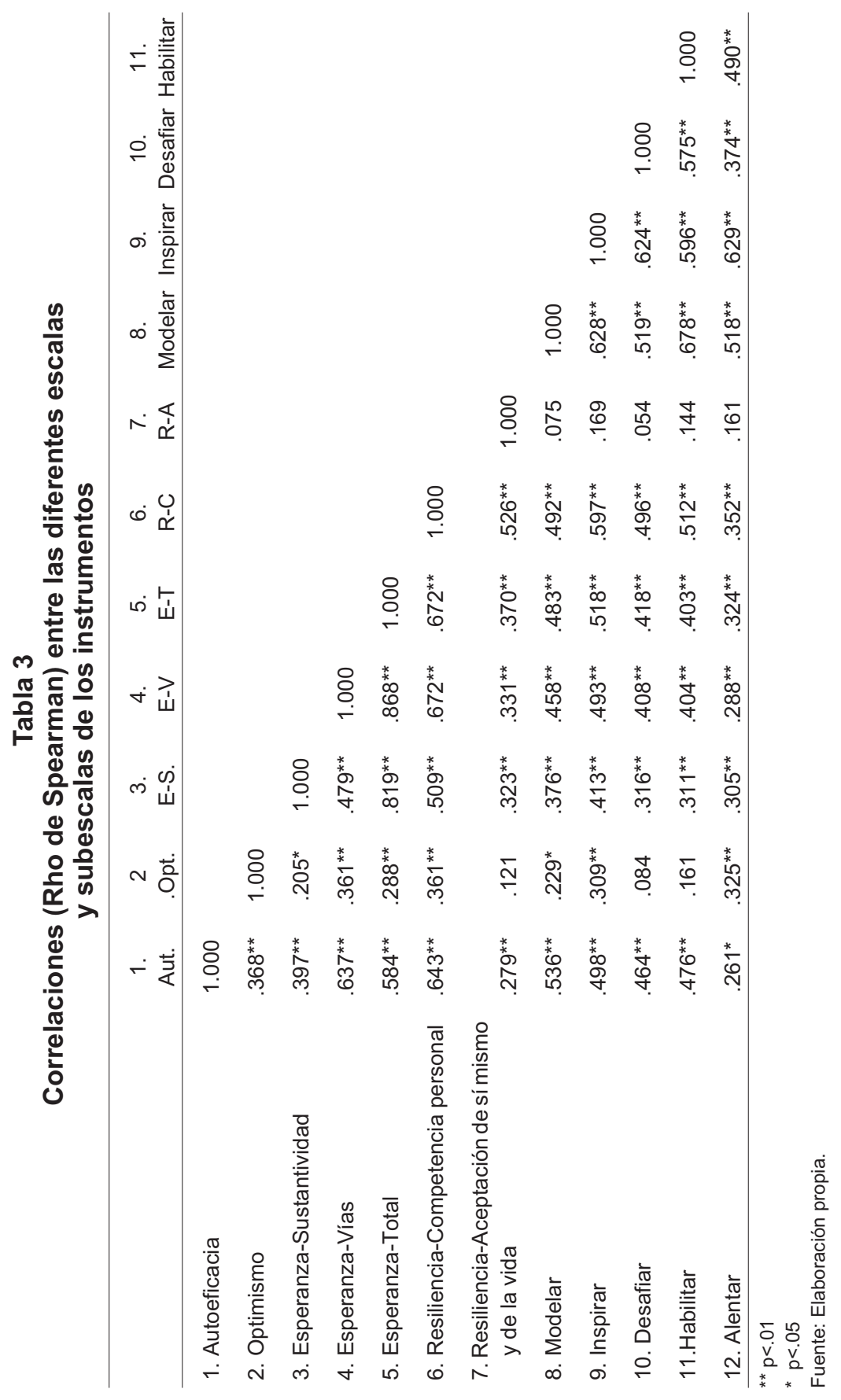


$\mathrm{do}=214.446, \mathrm{p}=.000)$ con una varianza explicada del $90 \%$. En el modelo 4 (Variable dependiente del IPL: Habilitar) la regresión resultó significativa (Chi cuadrado=203.393, $p=.000$ ) con una varianza explicada del $89 \%$. En el modelo 5 (Variable dependiente del IPL: Alentar) la regresión resultó significativa (Chi cuadrado=24.564, $p=.000$ ) explicando una varianza del $23 \%$.

En general, se observa en los modelos un buen ajuste con una varianza explicada elevada. Sin embargo, en el modelo 5 (Alentar) la varianza explicada es reducida, lo que indica que no todas las dimensiones del capital psicológico tienen el mismo poder explicativo sobre todas las prácticas del liderazgo.

En la misma Tabla 4, se muestran los coeficientes significativos de los diferentes modelos, el coeficiente $B$, constitu- ye el efecto de la variable independiente sobre la variable dependiente, al lado del mismo se observa su significación.

En esta tabla se observa la influencia de la resiliencia, en todas las prácticas de liderazgo, si bien de manera más reducida en la de alentar, también se observa la influencia de la percepción de autoeficacia en las prácticas de modelar, desafiar y habilitar, así como la influencia de las dimensiones de la esperanza, distribuida entre distintas prácticas de liderazgo. Finalmente no existe influencia del optimismo en ninguna de las prácticas de liderazgo.

Los coeficientes de la tabla son, en general, reducidos, especialmente en el caso de la Resiliencia, lo que demuestra que dicha influencia aunque existente es algo débil. Únicamente, coeficientes como los de la Autoeficacia, o los de las dimen-

Tabla 4

Modelos de regresión sobre las subescalas del IPL*

\begin{tabular}{|c|c|c|c|c|c|c|c|c|c|c|}
\hline & \multicolumn{2}{|c|}{$\begin{array}{l}\text { Modelo } 1 \\
\text { Modelar }\end{array}$} & \multicolumn{2}{|c|}{$\begin{array}{c}\text { Modelo } 2 \\
\text { Inspirar }\end{array}$} & \multicolumn{2}{|c|}{$\begin{array}{l}\text { Modelo } 3 \\
\text { Desafiar }\end{array}$} & \multicolumn{2}{|c|}{$\begin{array}{l}\text { Modelo } 4 \\
\text { Habilitar }\end{array}$} & \multicolumn{2}{|c|}{$\begin{array}{c}\text { Alentar } \\
\text { B }\end{array}$} \\
\hline EAG Total & 0.167 & ** & & & 0.096 & * & 0.128 & $* *$ & & \\
\hline \multicolumn{11}{|l|}{ Optimismo } \\
\hline \multicolumn{11}{|l|}{ Esperanza } \\
\hline Acción & & & 0.281 & ** & 0.124 & * & & & 0.165 & ** \\
\hline Vías de acción & & & & & & & & & -0.124 & * \\
\hline \multicolumn{11}{|l|}{ Resiliencia } \\
\hline Competencia personal & 0.059 & ** & 0.078 & ** & 0.083 & ** & 0.088 & ** & 0.048 & * \\
\hline $\begin{array}{l}\text { Aceptación de sí } \\
\text { mismo y de la vida }\end{array}$ & -0.053 & * & -0.059 & * & -0.056 & * & -0.074 & $* *$ & & \\
\hline
\end{tabular}

${ }^{*} p \leq .05$

${ }^{* *} p \leq .01$

*solo se muestran los coeficientes significativos

Fuente: Elaboración propia. 
siones de la escala de Esperanza, muestran valores ligeramente más elevados.

En relación a las hipótesis planteadas en el estudio, se confirma la primera de las hipótesis, la cual indica que el capital psicológico de los directivos influye en sus prácticas de liderazgo. Esto es cierto, pero requiere de alguna matización, debido al efecto diferencial ejercido sobre las distintas prácticas de liderazgo, ya que la varianza explicada en el último modelo (Tabla 3) fue reducida, únicamente el $23 \%$. Así, el efecto puede ser mayor o menor, de manera general, dependiendo de la práctica a la que se aluda.

Por otra parte, si bien en general se confirma la segunda hipótesis, en la cual se indica que los componentes independientes del capital psicológico ejercen efectos sobre las prácticas de liderazgo, esto no es cierto en su totalidad, debido a la ausencia de influencia de la variable de optimismo. Esta falta de influencia, así como el hecho de que no todas las dimensiones del capital psicológico influyen en todas las prácticas de liderazgo, hace que esta hipótesis sea solo parcialmente válida.

Al observar en su conjunto las características del capital psicológico de los directivos de PYMES que participaron en el estudio, se puede apreciar un alto nivel de capital psicológico, el cual se caracteriza de mayor a menor, por esperanza, resiliencia (orientada más a la competencia personal), autoeficacia y optimismo.

La variable Esperanza, obtuvo la puntuación más alta, tanto en su dimensión de generar alternativas para lograr (vías de acción) como en la determinación y la motivación suficiente para actuar (acción) (Snyder et al., 1991), esto es, pensamientos dirigidos hacia la acción que le permitirá al individuo cumplir sus metas. Este aspecto es relevante, en cuanto al parecer éstos directivos no solo buscan alternativas sino que guían su acción para alcanzar las metas que se proponen. Esta variable es especialmente importante si se tiene en cuenta que solo el $11.7 \%$ de los participantes son empleados dependientes, los demás o son empresarios o son independientes. Se sugiere realizar estudios para comprender el efecto de ésta variable sobre el emprendimiento, más aún si se tiene en cuenta que la esperanza en sus dos dimensiones está orientada hacia metas.

De forma coherente, el segundo componente más importante del Capital Psicológico de estos directivos fue la autoeficacia, o confianza en sí mismo, la cual hace referencia a la convicción de un individuo acerca de sus habilidades para movilizar la motivación, los recursos cognitivos y las acciones necesarias para lograr sus objetivos (Stajkovic y Luthans, 1998). Estos resultados, unidos con los obtenidos en la variable anterior, refuerza el efecto de los aspectos autorreferenciales en la orientación del individuo hacia el desarrollo de las metas propuestas.

Otro componente importante del capital psicológico de los participantes fue la resiliencia, variable en la que se obtuvo una puntuación total de 150.2 la cual es considerada alta de acuerdo con el instrumento utilizado (puntuación mayor a 147). Cabe anotar que la dimensión de la resiliencia más relevante en éste grupo de directivos, fue la orientada hacia la competencia personal, entendida como el reconocimiento de la capacidad personal, independencia, dominio, perseverancia, entre otros, más que hacia la 
aceptación de sí mismo y de la vida (segunda dimensión que evalúa el instrumento). Estos datos muestran la importancia de estudiar la resiliencia en relación con el liderazgo, principalmente desde la dimensión de las competencias.

Finalmente se sugiere estudiar el rol del optimismo en el capital psicológico de este grupo de directivos, pues aunque no puede ser considerado bajo, fue una de las variables menos destacadas. No es claro si los resultados obtenidos en este estudio respecto al optimismo obedezcan a su escasa relación con el liderazgo o a debilidades del constructo en sí mismo. En esta investigación se concibió como una atribución relativamente estable y global con respecto a eventos positivos (Seligman, 1998). Quizás deba estudiarse con mayor profundidad este concepto a fin de contribuir a la consolidación del constructo, pues en éste estudio no solo obtuvo la menor puntuación sino que su efecto en general sobre las variables estudiadas fue débil.

Con respecto a las prácticas de liderazgo el modelar el camino y habilitar a los demás para actuar obtuvieron las puntuaciones más altas (26.16 y 25.34 respectivamente), ambas características del liderazgo transformacional. En tercer lugar se situó el Alentar al Corazón (24.49) considerada característica del liderazgo transaccional. Las otras dos variables, pertenecientes al liderazgo transformacional aunque fueron más bajas no distan mucho de las anteriores, la que puntuó menos fue la de inspirar. Estos resultados confirman que el liderazgo transformacional y el transaccional no constituyen categorías excluyentes, pudiéndose presentar ambas simultáneamente.
Tal como aparece en la literatura, las prácticas de liderazgo transformacional, característico de éste grupo de directivos suele presentarse en líderes que poseen en su conjunto, éstas cuatro características, de Capital Psicológico (Toor y Ofori, 2010), lo cual fue evidente en éste estudio cuyos resultados apoyan la idea de que las variables del capital psicológico deben ser vistas como un continuo, y que éstas, en su conjunto, configuran el constructo, tal como lo plantean Avey et al. (2008). Así mismo se encontró correlación entre las prácticas de liderazgo y las variables de capital psicológico, salvo en la de resiliencia orientada hacia la aceptación de la vida y de sí mismo, nuevamente se confirma la pertinencia de estudiar la resiliencia en el liderazgo pero particularmente desde la competencia personal y no desde aspectos como aceptación de sí mismo y de la vida.

En este estudio, las variables del capital psicológico se relacionaron de manera significativa con las prácticas de liderazgo (aunque de forma moderada), no obstante, no hubo ninguna relación con la resiliencia, en la dimensión aceptación de la vida y de sí mismo quizás porque es un aspecto más de carácter intraindividual.

De acuerdo con los modelos de regresión utilizados, el capital psicológico si influye en las prácticas de liderazgo, solo que su nivel de impacto es diferencial, esto es, su capacidad de explicar cada una de las prácticas fue diferente. Esto es relevante debido a que el capital psicológico de los líderes y de los seguidores también se ha relacionado con aspectos de rendimiento (Walumbwa, Peterson, Avolio y Hartnell, 2010), si bien la relación, en el caso de los seguidores, está mediada por aspectos de clima laboral. 
La autoeficacia, la esperanza y la resiliencia ejercen influencia sobre las prácticas de liderazgo, de forma distinta en cada una de ellas. De acuerdo con estos resultados, estas variables del capital psicológico parecen tener un mayor efecto sobre las prácticas de liderazgo, mientras que el optimismo al parecer no ejerce ninguna influencia.

A pesar de que los coeficientes no fueron muy elevados, son suficientes para aceptar la hipótesis de que el capital psicológico influye en las prácticas de liderazgo, pero no de la misma manera, al parecer hay un efecto diferencial que requiere ser estudiado. Lo anterior puede corroborarse en la segunda hipótesis que se acepta parcialmente, pues aunque de manera independiente la autoeficacia, la resiliencia y la esperanza afectan las prácticas de liderazgo, el optimismo no la ejerce, se sugiere en futuros estudios estudiar esta variable utilizando otros modelos de liderazgo. El capital psicológico ha sido encontrado también, en otros estudios, el componente principal influenciando el liderazgo (Jensen y Luthans, 2006).

\section{Conclusiones}

Los directivos de las PYMES que participaron en el estudio presentaron un nivel alto de capital psicológico, caracterizado principalmente por la esperanza, el cual se asocia a su vez con la persistencia y se manifiesta en su capacidad para comenzar y mantener cursos de acción necesarios para alcanzar sus objetivos. De acuerdo con los resultados de este estudio, la capacidad de emprendimiento puede estar relacionada con esta variable, en cuanto un alto porcentaje de parti- cipantes son empresarios/independientes. De acuerdo con esto, se sugiere desarrollar investigaciones al respecto.

De acuerdo con las prácticas de liderazgo evaluados, los directivos de PYMES colombianas presentan un liderazgo transformacional con algunas características transaccionales, lo que confirma la existencia de un perfil de liderazgo, que no excluye prácticas pertenecientes a otras categorías.

En este estudio se observó que las prácticas de liderazgo se relacionan con el capital psicológico y que éste último puede influir en el tipo de liderazgo ejercido.

Se sugiere continuar estudiando el capital psicológico en relación con el liderazgo, pues aunque en esta investigación se verifica la influencia del capital psicológico sobre las prácticas de liderazgo, este efecto se da de forma diferencial, lo cual requiere de una mayor indagación acerca de las causas de la misma.

\section{Referencias bibliográficas}

Avey, James; Luthans, Fred y Jensen, Susan (2009). "Psychological capital: A positive resource for combating employee stress and turnover". Human Resource Management, Vol. 48 No. 5, p. 677-693.

Avey, James; Luthans, Fred y Mhatre, Ketan (2008). "A call for longitudinal research in positive organizational behavior". Journal of Organizational Behavior, Vol. 29, p. 705-711.

Avey, James; Luthans, Fred y Youssef, Carolyn (2010). "The Additive Value of Positive Psychological Capital in Predicting Work Attitudes and Behaviors". Journal of Management, Vol. 36 No. 2, p. 430-452. 
Baessler, Judith y Schwarzer, Ralf (1996).

"Evaluación de la autoeficacia: Adaptación española de la escala de autoeficacia general". Ansiedad y estrés, Vol. 2 No. 1, p. 1-8.

Bratt, Marlyn; Broome, Marion; Kelber, Sheryl y Lostocco, Lynne (2000). "Influence of stress and nursing leadership on job satisfaction of pediatric intensive care unit nurses". American Journal of Critical Care, Vol. 9, No. 5, p. 307317.

Chiok Foong Loke, Jennifer (2001). "Leadership behaviors: Effects on job satisfaction, productivity and organizational commitment". Journal of Nursing Management, Vol. 9, p. 191-204.

Clapp-Smith, Rachel; Vogelgesang, Gretchen y Avey, James (2009). "Authentic Leadership and Positive Psychological Capital: The Mediating Role of Trust at the Group Level of Analysis". Journal of leadership y Organizational Studies, Vol. 15, No. 3, p. 227-240.

Dunham-Taylor, Janne. (2000). "Nurse executive transformational leadership found in participative organizations". Journal of Nursing Administration, Vol. 30, No. 5, p. 241-250.

Eisenberger, Robert; Fasolo, Peter y Davis-LaMastro, Valerie (1990). "Perceived organizational support and employee diligence, commitment, and innovation". Journal of Applied Psychology, Vol. 75, p. 51-59.

Feldman, David y Snyder, Charles (2000). The State Hope Scale. En John Maltby, Christopher Alan Lewis, y Andrew Hill (Eds.), A handbook of psychological tests (pp. 240-245). Lampeter, Wales: Edwin Mellen Press.

Ferrando, Pere; Chico, Eliseo y Tous, Josep (2002). "Propiedades psicométricas del Test de Optimismo. Life Orientation Test". Psichotema, Vol. 14, p. 673-680.
Gooty, Janaki; Gavin, Mark; Johnson, Paul; Frazier, Lance y Snow, Bradley (2009). "In the Eyes of the Beholder: Transformational Leadership, Positive Psychological Capital, and Performance". Journal of Leadership y Organizational Studies, Vol. 15, No. 4 , p. 353-367.

Heilemann, Mary Sue; Lee, Kathryn y Kury, Felix (2003). "Psychometric Properties of the Spanish Version of the Resilience Scale". Journal of Nursing Measurement, Vol. 11, No. 1, 61-72.

Jerusalem, Matthias y Schwarzer, Ralf (1992). Self-efficacy as a resource factor in stress appraisal processes. En Ralf Schwarzer (Ed.). Self-efficacy: Thought control of action (pp.195213). Washington, D.C, USA: Hemisphere.

Jiayan, Liu; Oi-Ling, Siu y Kan, Shi (2010). "Transformational Leadership and Employee Well-Being: The Mediating Role of Trust in the Leader and Self-Efficacy". Applied Psychology: An international Review, Vol. 59, No. 3, p. 454-479.

Juarez, Fernando y Contreras, Francoise (2008). "Psychometric properties of the General Self-efficacy Scale in a Colombian Sample". International Journal of Psychological Research, Vol. 1, No. 2, p. 6-12.

Kouzes, James y Posner, Barry (1988). "The Leadership Practices Inventory". San Diego, CA: Pfeiffer.

Kouzes, James y Posner, Barry (2003). "The leadership Practices Inventory (LPI): Self Instrument". San Francisco: Jessey-Bass/ Pfeiffer.

Luthans, Fred y Youssef, Carolyn (2004). "Human, Social, and Now Positive Psychological Capital Management: Investing in People for Competitive Advantage". Organizational Dynamics, Vol. 33, No. 2, p. 143- 160. 
Luthans, Fred (2002). "The need for and meaning of positive organizational behavior". Journal of Organizational Behavior, Vol. 23, p. 695-706.

Luthans, Fred; Avolio, Bruce; Avey, James y Norman, Steven (2007). "Positive psychological capital: Measurement and relationship with performance and satisfaction". Personnel Psychology, Vol. 60, p. 541-572.

Luthans, Fred; Avolio, Bruce; Walumbwa, Fred y Li, Weixing (2005). "The psychological capital of Chinese workers: Exploring the relationship with performance". Management and Organization Review, Vol. 1, p. 247-269.

Luthans, Fred; Luthans, Kyle y Luthans, Brett (2004). "Positive psychological capital: Beyond human and social capital". Business Horizons, Vol. 47, No. 1, p. 45-50.

Luthans, Fred; Norman, Steven; Avolio, Bruce y Avey, James (2008). "The mediating role of psychological capital in the supportive organizational climate employee performance relationship". Journal of Organizational Behavior, Vol. 29, p. 219-238.

Luthans, Fred; Youssef, Carolyn y Avolio, Bruce (2007). "Psychological capital". Oxford, UK: Oxford University Press.

McNeese-Smith, Donna (1999). "The relationship between managerial motivation, leadership, nurse outcomes and patient satisfaction". Journal of Organizational Behavior, Vol. 20, No. 2, p. 243-259.

Mendoza, Ignacio (2005). "Estudio diagnostico del perfil de liderazgo transformacional y transaccional de gerentes de ventas de una empresa farmacéutica a nivel nacional". Tesis de doctorado no publicada, ciencias económicas y administrativas, Universidad Autónoma de Tlaxcala.
Norman, Steven; Avey, James; Nimnicht, James y Pigeon, Nancy (2010)."The Interactive Effects of Psychological Capital and Organizational Identity on Employee Organizational Citizenship and Deviance Behaviors". Journal of Leadership y Organizational Studies, Vol. 17, No. 4, p. 380-391.

Peterson, Suzanne y Luthans, Fred (2003). "The positive impact of development of hopeful Leaders". Leadership and Organization Development Journal, Vol. 24, p. 26-31.

Pirela, Ligia (2010). "Liderazgo y cultura organizacional en instituciones de Educación Básica". Revista Venezolana de Gerencia, Vol. 15, No. 51, p. 486-503.

Robles, Victor; de la Garza, María Isabel y Medina, José (2008).El liderazgo de los gerentes de las PYMES de Tamaulipas, México, mediante el Inventario de Practicas de Liderazgo. Cuadernos de Administración, Vol. 21, No. 37, p. 293-310.

Scheier, Michael; Carver, Charles y Bridges, Michael (1994). "Distinguishing optimism from neuroticism and trait anxiety, self-mastery, and self-esteem: A Reevaluation of the Life Orientation Test". Journal of Personality and social Psychology, Vol. 67, No. 6, p. 1063-1078.

Schwarzer, Ralf; Baessler, Judith; Kwiatek, Patricia; Schroder, Kerstin y Zhang, Jian (1997). "The assessment of optimistic self-beliefs: Comparison of the German Spanish and Chinese versions of the general self-efficacy Scale". Applied Psychology. An International Review, Vol. 46, No. 1, p. 69-88.

Seligman, Martin y Csikszentmihalyi, Mihaly (2000). "Positive psychology". American Psychologist, Vol. 55, p. 5-14.

Seligman, Martin (1998). "Learned optimism: How to change your mind and your life". New York, NY: Pocket Books. 
Snyder, Charles (1994). "The psychology of hope: You can get there from here". New York: Free Press.

Snyder, Charles (2000). "The past and possible future of hope". Journal of Social and Clinical Psychology, Vol. 19, p. 11-28.

Snyder, Charles; Harris, Cheri; Anderson, John; Holleran, Sharon; Irving, Lori; Sigmon, Sandra et al. (1991). "The will and the ways: Development and validation ofan individual-differences measure of hope". Journal of Personality and Social Psychology, Vol. 60 , p. $570-585$.

Snyder, Charles; Sympson, Susie; Ybasco, Florence; Borders, Tyrone; Babyak, Michael y Higgins, Raymond (1996). "Development and validation ofthe State Hope Scale". Journal of Personality and Social Psychology, Vol. 70, p. 321-335.

Sosik, John y Godshalk, Veronica (2000). "Leadership styles, mentoring functions received, and job related stress: a conceptual model and preliminary study". Journal of Organizational Behavior, Vol. 21, p. 365-390.

Sparks, John y Schenk, Joseph (2001). "Explaining the effects of transformational leadership: an investigation of the effects of higher-orders motives in multilevel marketing organizations". Journal of Organizational Behavior, Vol. 22, p. 849-869.

Stajkovic , Alexander y Luthans, Fred (1998). "Self-efficacy and work related perfor- mance: a meta-analysis". Psychological Bulletin, Vol. 124, p. 240 - 261.

Toor, Shamas-ur.Rehman y Ofori, George (2010). "Positive Psychological Capital as a Source of Sustainable Competitive Advantage for Organizations". Journal of Construction Engineering y Management, Vol. 136, No. 3, p. 341-352.

Upenieks, Valda (2003). "Nurse leaders' perceptions of what comprises successful leadership in today's acute inpatient environment". Nursing Administration Quarterly, Vol. 27, No. 2, p. 140-52.

Wagnild, Gail y Young, Heather (1993). "Development and psychometric evaluation of the Resilience Scale". Journal of Nursing Measurement, Vol. 1, p. 165-178.

Walumbwa, Fred; Luthans, Fred; Avey, James y Oke, Adegoke (2011). "Authentically leading groups: The mediating role of collective psychological capital and trust". Journal of Organizational Behavior, Vol. 32, No. 1, p. 4-24.

Walumbwa, Fred; Peterson, Suzanne; Avolio, Bruce y Hartnell, Chad (2010). "An Investigation of the Relationships among Leader and Follower Psychological Capital, Service Climate, and Job Performance". Personnel Psychology, Vol. 63, No. 4, p. 937-963.

Youssef, Carolyn y Luthans, Fred (2007). "Positive organizational behavior in the workplace: The impact of hope, optimism, and resiliency". Journal of Management, Vol. 33, p. 774-800. 